\title{
QoM and Lifetime-constrained Random Deployment of Sensor Networks for Minimum Energy Consumption
}

\author{
Morteza Maleki \\ Dept. of EE-Systems, University of Southern California, \\ Los Angeles, CA 90089 \\ morteza@usc.edu
}

\begin{abstract}
We consider the problem of energy efficient random deployment of sensor network. Our goal is to find the sensor node density, or alternatively, the energy resource density at every point inside a given deployment region, which results in allocating the minimum total number of deployed sensors, or alternatively, the minimum total energy source subject to constraints on the Quality of Monitoring (QoM) and network lifetime. The QoM is defined as the average of spatial distortion in reconstructed signal at the base station and can be bounded for a random deployment of sensor nodes when sensors are points of a Poisson process in the deployment region. To solve the optimization problem, we first determine a node density which satisfies the QoM constraint. Next we present a continuous space model for random deployment with the associated routing scheme that can be used to provide the minimum total required energy consumption. Finally, we present a spatial distribution of the sensor nodes (or the energy resources) that can achieve this minimum total energy. Simulation result shows that the minimum total energy obtained is close to the actual energy required in a randomly deployed dense network.
\end{abstract}

Keywords - Energy-awareness, Sensor networks, Mathematical programming/optimization, Random deployment.

\section{INTRODUCTION}

Sensor nodes can be deployed to monitor a phenomenon of interest in an area. These sensor nodes collect a variety of data, such as sound, motion, temperature, or vibrations and pass this data up toward a base station whose goal is to perform a high level monitoring tasks such as detecting seismic activity or securing some region. Sensor nodes tend to be severely constrained by the amount of battery energy that is available to them. This limitation in turn greatly impacts the service lifetime and the QoM that can be achieved by each sensor node or by the sensor network as a whole. A sensor network derives its strength from the rather large number of sensors that is includes. This brings to mind a variety of design and scale issues. Chief among these problems are those of sensor node deployment and energy resource allocation. The first problem is that of assigning a sensor node density, $\lambda$, to the deployment region whereas the second question relates to that of allocating the amount of initial energy, $e_{i}$, to each sensor. Assuming a fixed initial energy level, $e_{0}$, the total energy allocated to the network in a region $\mathcal{R}$ with area $\|\mathcal{R}\|$ is thus $e_{\text {tot }}=\lambda\|\mathcal{R}\| e_{0}$. In other words, for a fixed total energy bound $e_{t o t}$ in a fixed area $\mathcal{R}$, the parametric solutions $\left(\lambda \alpha, \alpha, e_{0}\right)$ for any $\alpha \gg 0$ are completely equivalent. Furthermore, $\alpha$ may change in different sub-regions (it can spatially vary.) Therefore, in the remainder of this paper, we will treat the sensor deployment problem (i.e., determining $\lambda$ ) and the energy allocation problem (i.e., setting $e_{i}$ per node) as related and

\author{
Massoud Pedram \\ Dept. of EE-Systems, University of Southern California, \\ Los Angeles, CA 90089 \\ pedram@ceng.usc.edu
}

interacting problems.

Deployment in practical sensor networks is usually random or, at best, controlled at a coarse granularity e.g., through aerial deployment. That is why when we refer to sensor node deployment, we avoid referring to the exact coordinates of individual sensors in the network. Instead we assume that the coarse-grain deployment mechanisms can only guarantee a random distribution with known density function. ${ }^{1}$ The problem that we address here is that of finding $\left(\lambda_{i}, e_{i}\right)$ that minimize $\int_{\mathcal{R}} \lambda_{i} e_{i}$ subject to constraints on QoM and network lifetime. Here index $i$ identifies some subregion $A_{i}$ in $\mathcal{R}$. The minimum size of $A_{i}$ is determined by the granularity of the deployment control. For example, if there is sensor deployment through ground vehicles $A_{i}$ is in the range of tens of meters whereas for the aerial sensor deployment is in the range of hundreds of meters. In our solution technique, we will assume that we have full control over node densities within some thin circular regions. Note that the situation is different after the network is deployed and has become operational since at that point the approximate locations of all sensors in the network can be determined and exploited by the scheduler.

The remainder of this paper is organized as follows. Section 2 describes the relevant work to this paper. In section 3 , we state the problem setup, definitions, objective and optimization problem. Section 4 presents optimum routing algorithms in a continuous space model of random deployment.

\section{RELATED WORK}

Many of the requisite features of a sensor network such as connectivity and coverage are significantly affected by the deployment strategy. Hence, sensor deployment strategies play a significant role in determining the performance of static sensor networks. To this end, there have been a number of studies that address this problem as explained in the following discussion.

Adlakha et al [1] study the factors of accuracy, latency and lifetime as design time optimization constraints with respect to node density. These factors are subject to change when some nodes fail or die out and as a result node density decreases. The lifetime of the network is defined as the network utility, which is computed as the product of the network's connectedness with the accuracy. The aim is

\footnotetext{
${ }^{1}$ We realize that having complete control over how the node density varies over space is in the limit equivalent to having precise control over the coordinates of individual sensors. However, we assume that there is a sufficient level of deployment control to allow for slowly varying node densities across space.
} 
to derive design parameters for the network that can be tuned by a cluster management scheme, as suggested by the authors as suggested by the authors in a previous work.

There have been a couple of research results on deriving an upper bound on the service lifetime of sensor networks. The service lifetime of a sensor network has been derived in [2][3] by multi commodity flow non-linear programming formulation. It can be shown that the problem we will state later in this paper which is finding minimum total energy (or total number of nodes) subject to the constraint on network lifetime is dual of finding the maximum network lifetime subject to constraint on the initial energy budget.

The work presented in [4] has tackled the optimization problem of sensor placement with constraints on distortion of the reconstructed sensed signal at the base and with the objective of minimizing the total transmission power for a given number of sensor nodes. This reference provides solutions for sensor placement on a line and a special case of sensor placement in a plane assuming that number of sensors is given and the sensors can be precisely placed at their desirable locations. Our paper extends this work to the case of sensor deployment in a plane where, because of lack of precise control in the deployment phase, the sensor locations are random although the sensor density can be controlled.

GAF [5], which uses intelligent node scheduling techniques to conserve the energy, is an example of energy conservation techniques that rely on node clustering and network routing. GAF divides the coverage area where the nodes are distributed to small virtual grid cells such that at any instant, only one node in each cell is active while all other nodes in that cell are in their power saving mode (sleep or discovery). After a node remains active for a period of time, it changes its state to power saving mode in order to give a chance to other nodes within the same cell to become active. We will show in our deployment scheme how to apply a proper duty cycle for turning on/off the nodes distributed in a ring such that all the nodes in the ring expire at the same time.

The abovementioned works are the most relevant works to our proposed paper. There is a large body of other works that address the connectivity [6] and coverage problems [6][7][8] in distributed sensing for generic applications (e.g., target tracking). Most of these works use uniform deployment strategy or a deployment scheme that minimizes the probability of detection along the exposure paths in the case of target tracking.

\section{PROBLEM SETUP}

We assume that boundaries of a sensor deployment region (or monitoring region) $\mathcal{R}$ and location of a sink node is known. The sink node gathers information from the monitoring region and has direct connection to a base station. We wish to calculate the value of a particular parameter, $\pi$, related to some phenomenon of interest in region $\mathcal{R}$ by frequently monitoring the region with a number of sensor nodes appropriately deployed in that region. Each sensor node frequently senses the environment and measures a value $Z$ at its present location or in its local environment (the size of this local environment is determined based on the sensing range of the sensor.) Next it reports its measurement by sending the quantized values of $Z$ to the designated sink node by using a (possibly) multiple-hop routing scheme. The reporting can be periodic or aperiodic. In periodic reporting a sensor node periodically reports its measurement to the base. However, in aperiodic monitoring a sensor reports its measurement when the value of $Z$ at its location or its sensing range exceeds a threshold, which indicates occurrence of an event at the local environment of the sensor. In either periodic or aperiodic reporting we work with the time average of the data rate for reporting $\mu$. and we assume that $\mu$. is constant for all sensor nodes inside the monitoring region.

The gathered information is then sent to the base, which subsequently aggregates the received sensor data. The notion of sensing range has different definitions depending on the task for which the sensors are deployed. For network interdiction, or more generally event detection, applications, the sensing range of a sensor is the neighboring area of the sensor where an event of interest can be detected by that sensor. In the environmental monitoring application such as temperature reading, each sensor measures the exact value of $\pi$ at the point where the sensor is located. The value of $\pi$ at a point in $\mathcal{R}$ where no sensor is co-located is estimated from the measurements of sensors, which are spatially correlated to that point.

\section{A. Model of Random Deployment}

Sensor nodes can be deployed in several ways in a given deployment region. The level of control in deployment of sensor nodes sets the degree of randomness in the locations of nodes. In a fine-grained deployment, there is no randomness in placement of sensor nodes and sensor nodes can be exactly placed in any location within the deployment region. In practice, however, it is usually infeasible to devise a deployment strategy whereby each sensor is placed precisely at some location. Practical deployment in large sensor networks is usually random, or at best, can be controlled with coarse granularity. As a result, adopting a random deployment model with slowly varying node densities is more realistic.

Consider $\mathcal{R} \subset \square^{k}$ as the deployment region where $\square^{k}$ denotes the $k$-dimensional Euclidian space. For a sufficiently large region $R$ where a large number of sensor nodes have been distributed in the area, the distribution can be modeled as a Poisson distribution in $k$ dimensional space [9]. Let $\lambda$ denote the node density i.e., $\lambda=\frac{n}{\|R\|}$ where $n$ is the number of nodes and $\|\mathcal{R}\|$ denotes the area of $\mathcal{R}$. Furthermore, consider that region $\mathcal{R}$ is partitioned into $m$ disjoint sub-regions, $A_{1} \ldots A_{m}$. Now for each $A_{i} \subseteq \mathcal{R}$, let $N\left(A_{i}\right)$ denotes the number of sensor nodes in $A_{i}$. We will make use of the following two key assumptions:

- Node distribution over region $A_{i}$ is a Poisson distribution with mean $\lambda\left\|A_{i}\right\|$.

- $N\left(A_{1}\right) \ldots N\left(A_{m}\right)$ are independent random variables.

\section{B. QoM and Network Lifetime Definition}

The QoM is intended to represent the "similarity" between the actual values of some parameter of interest in $\mathcal{R}$ and the reconstructed values for the same parameter in the base. These values can be thought of as a random field in the space and time. Therefore, QoM should be analyzed in two respects: temporal and spatial accuracy. The temporal accuracy may be defined by the reporting period whereas the spatial accuracy may be defined by accuracy of spatial estimation of the value of interest at the locations where no true value is available at the reconstruction time.

The temporal part of QoM is represented by frequency of sampling. For the remainder of this analysis, we assume that the sampling frequency is equal to or greater than the Nyquist frequency that can fully track and/or reconstruct changes of any phenomenon of interest inside region $R$. so that the base station can fully reconstruct 
the changes in time. In other words, we will not worry about temporal distortion. The base should also be able to spatially reconstruct the value of interest or events in $\mathcal{R}$ within the required minimum QoM. In this paper, spatial distortion is the primary concern here. By reducing this distortion, "similarity" between the reconstructed value and actual value of the parameter of interest increases thereby, improving the QoM metric. More precisely, we define an average spatial distortion metric, $\operatorname{MSE}(\mathcal{R})$, as follows:

$$
\operatorname{MSE}(\mathcal{R})=\frac{1}{\|\mathcal{R}\|} E\left(\int_{x \in \mathcal{R}} E[(Z(x)-\tilde{Z}(x))]^{2} d x\right)
$$

where $Z(x)$ and $\tilde{Z}(x)$ represent the actual and reconstructed values of interests at point $x$ in region $\mathcal{R}$ respectively. The second expectation is taken with respect to distribution of sensor nodes in $\mathcal{R}$ which are points of Poisson process.

It is assumed that the network designer or application developer imposes an upper bound constraint, $D_{\min }$, on the distortion metric as follows:

$$
\operatorname{MSE}(\mathcal{R}) \leq D_{\min }
$$

One can easily estimate the value of $Z$ at any point in $\mathcal{R}$ by linearly combining readings from its $k$ nearest sensor nodes through a method known as ordinary kriging [18]. First let's define the covariance between any two points $x_{i}$ and $x_{j}, C\left(x_{i}, x_{j}\right)$, as follows:

$$
C\left(x_{i}, x_{j}\right)=E\left[Z\left(x_{i}\right) Z\left(x_{i}\right)\right]=C_{0} \exp \left(-\gamma\left|x_{i}-x_{j}\right|\right)
$$

where $C_{0}=C(x, x), \gamma$ is a constant factor and $\left|x_{i}-x_{j}\right|$ is the Euclidean distance between $x_{i}$ and $x_{j}$.

We assume that deployed sensors inside region $\mathcal{R}$ are points of a Poisson process with density parameter $\lambda$ (as described in the section $3.1)$, and that $C\left(x_{i}, x_{j}\right)$ values are given. Furthermore $\mathcal{R}$ is sufficiently large.

Theorem 1: When using the ordinary kringing method to estimates the value of parameter $\mathrm{p}$ at every point $x$ in region $\mathcal{R}, M S E(\mathcal{R})$ can be bounded as follows:

$$
\operatorname{MSE}(\mathcal{R}) \leq C_{0} \sqrt{\frac{\pi}{\alpha_{0}}} \exp \left(\frac{1}{4 \alpha_{0}}\right)\left(1-\operatorname{erf}\left(\frac{1}{2 \sqrt{\alpha_{0}}}\right)\right)
$$

where $\alpha_{0}=\frac{2 \cdot \pi \cdot \lambda}{\gamma^{2}}$.

All proofs are provided in technical report [11]. They are skipped here due to space limitation.

Notice that in deriving the above bound, we used $\mathrm{k}=1$ for the ordinary kriging method. Obviously, the MSE error is reduced as we increase $k$. In other words equation (2) provides an upper bound on the actual error. Theorem 1 gives a simple equation form to bound $\operatorname{MSE}(\mathcal{R})$. Simulation results presented in [11] show that this bound is sufficiently close to the measured value of $\operatorname{MSE}(\mathcal{R})$ for intermediate to large values of $\lambda$. Consequently, we replace constraint (1) with the following constraint:

$$
C_{0} \sqrt{\frac{\pi}{\alpha_{0}}} \exp \left(\frac{1}{4 \alpha_{0}}\right)\left(1-\operatorname{erf}\left(\frac{1}{2 \sqrt{\alpha_{0}}}\right)\right) \leq D_{\min }
$$

It can easily be shown that the bound is a monotonic decreasing function of $\lambda$, therefore, there is $\lambda_{\text {min }}$ such that:

$$
\operatorname{MSE}(\mathcal{R}) \leq D_{\min } \Leftrightarrow \lambda \geq \lambda_{\min }
$$

The sensor network lifetime is defined as the duration of time between when all sensor energy sources are replenished and when the spatial distortion in the reported value of interest in region $\mathcal{R}$ exceeds $D_{\min }$. Notice that the spatial distortion increases with time as some sensor nodes exhaust their energy source, and consequently, exit the ad hoc sensor network. Also notice that this distortion can rise slowly or rapidly over time, depending on the distribution of sensors inside the region coupled with how traffic is routed inside the network.

Quality and Lifetime-constrained Sensor Deployment Problem $(Q L S D):$ Given are boundaries of region $R$ and the location of the sink (base station), an upper bound, $D_{\text {min }}$ on average spatial distortion as a metric that represents QoM, and a lower bound, $T_{\text {min }}$, on the Network Lifetime. Assuming random deployment of sensors with the model presented in section 3.1, the objective is to find node density or energy density at each point in $\mathcal{R}$ so as to minimize the number of deployed nodes or the total energy allocated to the nodes in the network while satisfying the QoM and network lifetime constraints.

Key Assumptions: Before formulizing the QLSD problem we state a number of assumptions.

1. For sake of simplicity in calculation, we assume that $R$ is circular $\left(\mathcal{R} \subset \square^{2}\right.$ ) where the base is located at the center of the region. This case clearly encompasses the case where region $\mathrm{R}$ is a line.

2. Sensor nodes generate traffic at a constant data rate. They send their traffic with multi hop routing toward the sink. If there are spatial correlations between the readings of different sensor nodes, data compression is done at each source by a joint entropy coding method like Slepian-Wolf source coding [10]. As a result, there is no need for any data aggregation at intermediate nodes.

3. The MAC layer is ideal in the sense that there will be no energy loss due to the MAC layer collision. In addition, each node is scheduled to wake up only when required (i.e. when it wants to send or receive data.)At all other times, the sensor nodes are sleep, and hence, they do not dissipate any energy.

4. The sensor nodes are time-synchronized (see [13][14] for mechanisms to achieve time synchronization in a sensor network), meaning that all of the nodes that wakeup and sense their environment in some reporting cycle do so exactly at the same time instance. However, not every node is awakened in each cycle. More precisely, we assume that each sensor node is given a probability of wakeup and wakes up in a given cycle with that specified probability (cf. Section 4.2.)

5. Sensors can find their locations after they are deployed. They can use localization algorithms such as those in [15] to obtain their spatial coordinates with respect to the sink node. This information is then used by the sensors, who must also be knowledgeable about the deployment solution, to calculate and set their wakeup probabilities (cf. Section 4.2.) 
6. Wireless communication links between sensors are assumed to be lossless. In other words, no energy is dissipated for data retransmission due to the packet loss.

Modeling the Objective Function: Let $d x$ denote an infinitesimal subregion of $\mathcal{R}$ around point $x$ and $e(x)$ denotes the energy consumption rate of a node located at point $x$. Recall that $\mu$ is the average reporting rate. The total energy consumption in the network during time $T, e_{t o t}$, is:

$$
e_{t o t}=T \int_{\mathcal{R}} e(x) N(d x)
$$

where $N(d x)$ denotes the number of nodes in infinitesimal area $d x$. Now because of the properties of a Poisson point process:

$$
N(d x)=\left\{\begin{array}{l}
\geq 2 \text { with prob. }(\lambda d x)^{2} \approx 0 \\
1 \quad \text { with prob. } \lambda d x \\
0 \quad \text { with prob. } 1-\lambda d x
\end{array}, E[N(d x)]=\lambda d x .\right.
$$

The expected value of $e_{t o t}$ is:

$$
E\left[e_{t o t}\right]=T \int_{\mathcal{R}} E[e(x)] \lambda \mathrm{d} x
$$

Now we can write QLSD problem as a mathematical program:

$$
\begin{aligned}
& \operatorname{Min}_{e(x), \lambda, T} T \int_{\mathcal{R}} E[e(x)] \lambda \mathrm{d} x \quad \text { s.t. } \\
& C_{0} \sqrt{\frac{\pi}{\alpha_{0}}} \exp \left(\frac{1}{4 \alpha_{0}}\right)\left(1-\operatorname{erf}\left(\frac{1}{2 \sqrt{\alpha_{0}}}\right)\right) \leq D_{\text {min }} \\
& \text { where } \alpha_{0}=\frac{2 \pi \lambda}{\gamma^{2}} \\
& T \geq T \text { min }
\end{aligned}
$$

The objective function must be minimized with respect to three variables: $\lambda, T$ and $e(x)$. The objective function with respect to $\lambda$ and $T$ is linear. Moreover, it can be easily shown that there are $\lambda_{\min }$ and $T_{\min }$ that can satisfy constraints and minimize the objective with respect to $\lambda$ and $T$. Hence, we will focus on minimizing the objective with respect to $e(x)$ which is a function of the data routing algorithm in the sensor network.

\section{OPTIMAL ROUTING ALGORITHMS IN THE CONTINUOUS SPACE MODEL OF RANDOM DEPLOYMENT}

In this section we introduce a continuous-space model of random deployment of sensor networks and propose algorithms for data routing in that model such that objective function in (6) is minimized. We define an energy density, $e_{d}$ metric which captures the spatial distribution of energy resources inside area $\mathcal{R}$. As stated in Section 3, we assume that $R \subset \square^{2}$ is circular and the sink is located at the center of region (see Figure 1.)

\section{A. Continuous Space Model and Routing Algorithm}

Assume that each sensing event results in generation of B packets of data to be transmitted to the sink node. We can thus define an energy dissipation per packet of data for the sensing operation itself (denoted by $\alpha_{\mathrm{s}}$ ) and an energy per packet of data for delivering the data to the sink node (denoted by $w(x)$ ). The latter includes all of the energy consumptions related to receiving and/or sending the packet of data on its path from a point $x$ (i.e., $x=(r, \theta)$ in polar coordinates) to the sink. It can easily be shown that the total energy consumption, $e_{t o t}$, in the network during time $T_{\text {min }}$, and its mean value are:

$$
\begin{aligned}
& \mathrm{e}_{\mathrm{tot}}=T_{\min } \int_{\mathcal{R}}\left(\alpha_{s}+w(x)\right) \mu N(d x) \\
& E\left[e_{t o t}\right]=T_{\min } \int_{\mathcal{R}}\left(\alpha_{s}+E\left[w_{c}(x)\right]\right) \mu \lambda \mathrm{dx}
\end{aligned}
$$

Recall that $\mu$ is the average reporting rate. The above equation is in fact another equivalent form of the objective function in (6). Therefore, our model is a continuous space model wherein an infinitesimal area, $d x$, can be treated as source of traffic with local flow $\mu \lambda d x$. Furthermore, each source node must find a path to the sink, which minimizes total energy for sending its data to the sink. The conclusion is that Least-cost Path (LP) routing provides the optimal routes from each node to the sink in the QLSD problem.

For a first order radio model of a wireless sensor node [11], the transmission energy per bit $\varepsilon$ is related to the transmission distance $d$ as $\varepsilon=\alpha_{T 0}+\alpha_{T} d^{\beta}, \quad \beta>1$, where $\alpha_{T 0}$ and $\alpha_{T}$ are constants.

Let $S$ denote a source which is located at distance $r$ from the sink and assume that its traffic is relayed by $m-1$ intermediate sensors before reaching the sink. The work in [12] has proved that in this case when each hop has the same length (set to $r / m$ ), the total transmission energy is minimized. Therefore, the minimum energy for sending and relaying this packet to the sink is calculated as

$$
w(r)=m\left(\alpha_{T 0}+\alpha_{T}\left(\frac{r}{m}\right)^{\beta}\right)+(m-1) \alpha_{R} .
$$

where $\alpha_{R}$ denotes the energy cost for receiving a bit.

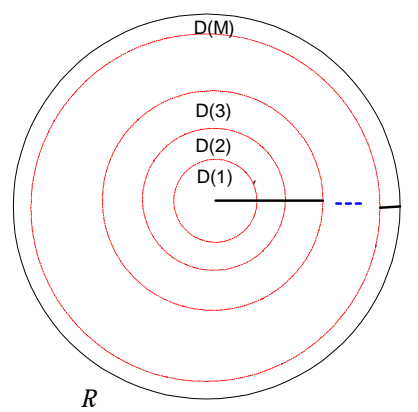

Figure 1. Deployment region is divided to discs wherein nodes within a disc have same hop-count to the sink.

By setting the derivative of $w(r)$ with respect to $m$ to 0 , we obtain $m *$ that minimizes $w(r)$ as follows:

$$
m^{*}=r / \sqrt[\beta]{(\beta-1) \alpha_{T} /\left(\alpha_{R}+\alpha_{T 0}\right)}
$$

Note that $w$ has only one extremum with respect to $m$ when $m>0$. Now, the optimal number of relaying hops should be an integer, i.e., either Int $\left[m^{*}\right]=\left\lfloor m^{*}\right\rfloor$ or $\left\lfloor m^{*}\right\rfloor+1$ and the distance of each relaying hop $d_{S}$ is constant and satisfies $d_{s}=r / \operatorname{Int}(m *)$. 


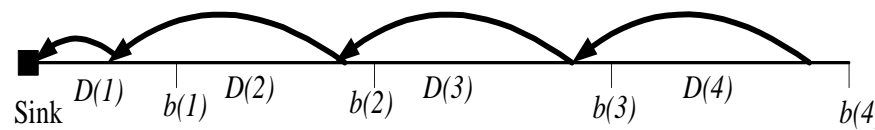

Figure 2. Optimal radial path for a node located in $D(4)$.

The optimal hop length of each sensor can be calculated iteratively starting from the center of the circular region along the radial line segment on which the sensor is placed. In other words, because of the perfect symmetry of the problem setup in the polar coordinates, the 2D sensor deployment problem in a circular region is transformed to a series of 1-D sensor deployments on different radial line segments (starting from the sink node which is at the center of the region and extending to the boundary of the circular region). Details are included in the Continuous Space Radial Transmission (CSRT) algorithm provided below. In this algorithm, $R$ denotes the length of the 1-D deployment region. Now consider a point $x$ which is at distance $r \leq R$ from the sink. We define $b(m)$ as the $r$ value which results in equalization of the minimum energy dissipation for forwarding a packet from $x$ to the sink by using exactly $m$ hops and that for forwarding the packet by using exactly $m+1$ hops. Notice that for all points $x$ that lie in the range $(b(m-1), b(m)]$, the minimum-energy hop length is $r / m$. From this definition and equation (7), we have:

$m\left(\alpha_{T 0}+\alpha_{T}\left(\frac{b(m)}{m}\right)^{\beta}\right)+(m-1) \alpha_{R}=(m+1)\left(\alpha_{T 0}+\alpha_{T}\left(\frac{b(m)}{m+1}\right)^{\beta}\right)+m \alpha_{R}$

The CSRT algorithm shows how the various $b(m)$ values are calculated for $m=1, \ldots, M$. Notice that line 4 is the solution of equation (9). Let the predecessor of a node located at $x=r$ be the node that is closer to the sink and who relays the data sent out from this node toward the sink. The CSRT algorithm assigns to each point $x=r$, a predecessor $x_{p}=r-r / m$ along the radius toward the sink.

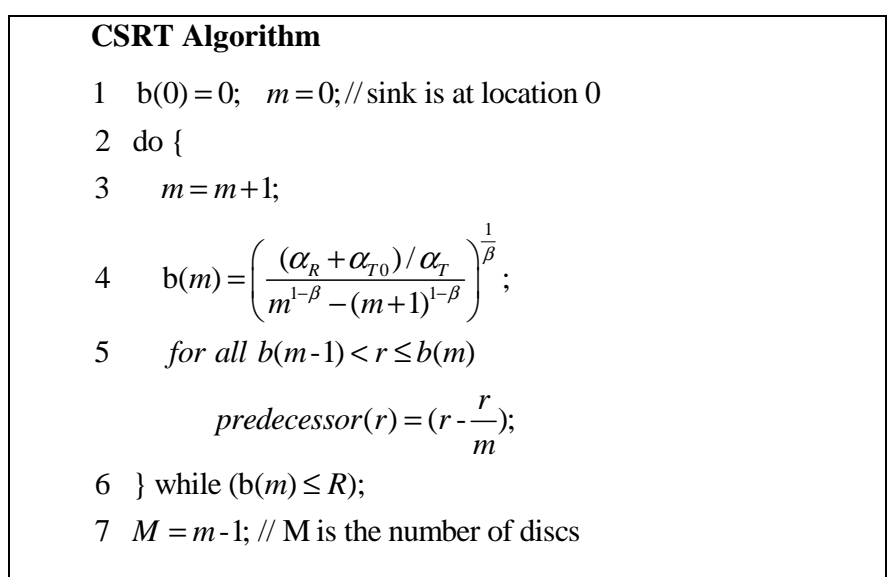

The CSRT algorithm (when applied to all radial lines with angle $\theta$ ) divides the 2-D deployment region, $\mathcal{R}$, to concentric disks $D(m)$ as shown in figure 1 where each disk, $D(m)$, specifies a subregion comprising of points $(r, \theta)$ as follows:

$$
\begin{aligned}
& D(m)=\{(r, \theta): b(m-1)<r \leq b(m) \text { and } 0 \leq \theta<2 \pi\} \\
& \text { for } 1 \leq m \leq M .
\end{aligned}
$$

Therefore, for each node $x=(r, \theta) \in D(m)$,its predecessor is $x_{p}=(r-r / m, \theta)$. Figure 2 is an example that shows how a node which is located inside $D(3)$ sends its packets toward the sink through the optimal path. All hops have equal length except for the last hop which connects to the sink. Optimality of the CSRT algorithm follows from the following lemmas and theorem.
Lemma 1: $b(m)$ (where $m \epsilon Z+)$ is an increasing function of $m$.

Lemma 2: For $m \geq 1$, if $b(m)<r \leq b(m+1)$, then $b(m-1)<r-r /(m+1) \leq$ $b(m)$.

Theorem 2: For a sensor located at $(r, \theta) \epsilon D(m)$, the predecessor in the Least-cost Path (LP) is a senor located at $(r-r / m, \theta) \epsilon D(m-1)$.

The proofs of the above lemmas and theorem can be found in [16].

\section{B. Energy Density and Total Energy}

Now we set out to determine the optimum energy density in $\mathcal{R}$ and the total energy allocated to the sensor nodes in the network under the condition that the CSRT algorithm is used for data routing.

Theorem 3: By employing the CSRT algorithm for data routing in the sensor network, energy density, $e_{d}(r)$, at point $(r, \theta)$ can be calculated as:

$e_{d}(r)=\left(\frac{(M+m-1+\chi)(M-m-2+\chi) \eta}{2 m}-\alpha_{R}+\alpha_{S}\right) \mu \lambda_{\min } T_{\min }$
$\forall(r, \theta) \in D(m)$ and $1 \leq m \leq M$ where $\chi=1_{\left(\frac{M r}{m} \geq R\right)}= \begin{cases}1 & \text { if } \frac{M r}{m} \geq R \\ 0 & \text { else }\end{cases}$

and $\eta=\alpha_{T}\left(\frac{r}{m}\right)^{\beta}+\alpha_{T 0}+\alpha_{R}$

Proof: To find flow distribution and as a result energy density at distance $r$ from the sink, the deployment region is divided into infinitesimally narrow rings around the sink with width $\Delta r$ (see figure 3.) We denote such a ring as $\rho(r)$ and define it as follows:

$$
\rho(r)=\left\{\left(r^{\prime}, \theta\right): r \leq r^{\prime}<r+\Delta r \text { and } 0 \leq \theta<2 \pi\right\}
$$

As stated earlier for the continuous space model, each infinitesimal area may be treated as a source of traffic with the local flow being proportional to the probability that one node is found in that infinitesimal area. Notice that from the Poisson process property, if a node is in $\rho(r)$, then it will have equal probability of residing in any location inside that $\rho(r)$. Therefore, the local flow of each $\rho(r)$ is uniformly distributed in the area of that ring. Therefore, the energy density inside the ring is uniform.

Let's define $M$ as the network diameter and let $\Delta I(r)$ and $\Delta F(r)$ denote total input/output flow to/from $\rho(r))$ and $\Delta L(r)$ denote locally generated flow in $\rho(r)$.

$$
\Delta L(r)=2 \pi r \Delta r \mu \lambda_{\min }
$$

By writing flow conservation for any $\rho(r)$ where $0 \leq r<R$, we get

$$
\Delta F(r)=\Delta L(r)+\Delta I(r) \text { and } \Delta I(r)=0 \text { if }(r, \theta) \in D(M)
$$

Notice that if $(r, \theta) \in D(m) \quad$ and $\quad l \leq m \leq M-2 \quad$ then $\left(\frac{m+1}{m} r, \theta\right) \in D(m+1)$ thus,

$$
\begin{aligned}
& \Delta F(r)=\Delta L(r)+\sum_{i=1}^{M-m-1} \Delta L\left(r+\frac{i r}{m}\right)+\Delta L\left(\frac{M r}{m}\right) \chi \\
& (r, \theta) \in D(m) \text { and } 1 \leq m \leq M
\end{aligned}
$$

The lifetime of a node that resides in $\rho(r)$, denoted by $\tau(r)$, can be calculated as follows: 
$\frac{2 \pi r \Delta r e_{d}(r)}{\left(\alpha_{T 0}+\alpha_{T}(r / m)^{\beta}\right) \Delta F(r)+\alpha_{R}(\Delta F(r)-\Delta L(r))+\alpha_{S} \Delta L(r)}=\tau(r)$

To fully utilize total energy allocated to all nodes in the deployment region and avoid energy waste, we want to ensure that all sensor nodes have the same lifetime, which is equal to the network lifetime, $T_{\min }$. Consequently, from the above equation, energy density, $e_{d}(r)$, is calculated as follows:

$e_{d}(r)=\frac{\left(\left(\alpha_{T 0}+\alpha_{T}(r / m)^{\beta}\right) \Delta F(r)+\alpha_{R}(\Delta F(r)-\Delta L(r))+\alpha_{S} \Delta L(r)\right) T_{\min }}{2 \pi r \Delta r}$

By substituting $\Delta \mathrm{F}(r)$ and $\Delta L(r)$ given by equations (12) and (13) in the above equation, we derive equation (11) and this complete the proof.

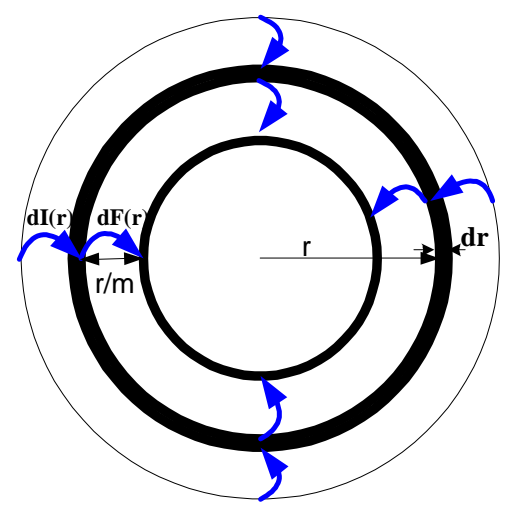

Figure 3. $d I(r)$ and $d F(r)$ are total input and output flows of an infinitesimally narrow ring with width $d r(\rho(\mathrm{r}))$ and $\mathrm{r} / \mathrm{m}$ is optimal hop-length for a sensor within the disc $D(m)$.

As explained earlier sensor deployment problem and the energy allocation problem are related and interacting problems. Indeed there are two ways to provide energy density for a $\rho(r)$. One way is to allocate $2 \pi r \Delta r e_{d}(r)$ to a node inside $\rho(r)$. However, if the battery capacity of each node is fixed to $E_{0}$ another way to allocate the required energy is to adjust node density inside $\rho(r)$ according to the following equation:

$\lambda_{d}(r)=\frac{e_{d}(r)}{E_{0}}=\left(\frac{(M+m-1+\chi)(M-m-2+\chi) \eta}{2 m}-\alpha_{R}+\alpha_{S}\right) \frac{\mu \lambda_{\min } T_{\min }}{E_{0}}$

for $(r, \theta) \in D(m)$ and $1 \leq m \leq M$

where $\eta=\alpha_{T}(r / m)^{\beta}+\alpha_{T 0}+\alpha_{R}$ and $\chi=1_{\left(\frac{M r}{m} \geq R\right)}$

This adjustment increases the node density above than the density $\lambda_{\min }$ that requires for satisfaction of QoM constraint. Therefore a policy must be employed to control the node density such that at any reporting cycle, density of active nodes which are participating in sensing the environment and data routing reduces to $\lambda_{\min }$ and the rest of the nodes are in the sleep mode (power saving).Therefore, not every node is awakened in each reporting cycle. More precisely, we assume that each sensor node is given a probability of wakeup and wakes up in a given cycle with that specified probability. That probability for a sensor which is located at distance $r$ from the sink is given as $\lambda_{\text {min }} / \lambda(r)$ (Note that $\lambda_{\text {min }} / \lambda(r) \leq 1$.)

The underlying assumption in CSRT and theorem 3 is that any point in the region can find an optimal radial path to the sink which consumes least energy according to equations (7) and (8). Therefore, CSRT derives minimum the total energy needs to be allocated to $R$.
Corollary 1: The minimum of the expected total energy required in the sensor network is:

$$
\begin{aligned}
& \left.E\left(e_{t o t}\right) \geq \lambda_{\min } \mu T_{\min } \sum_{m=1}^{M} \int_{r \in D(m)} 2 \pi r e(r) d r\right) \\
& =2 \pi \mu \lambda_{\min } T_{\min }\left(\frac{\alpha_{T}}{\beta+2} \sum_{m=1}^{M} \frac{\left(b^{\square}(m)\right)^{\beta+1}-\left(b^{\square}(m)\right)^{\beta+1}}{m^{\beta-1}}\right. \\
& \left.\left.+\frac{\alpha_{R}-\alpha_{S}}{2} \sum_{m=1}^{M}\left(b^{\square}(m)\right)^{2}-\left(b^{\square}(m)\right)^{2}\right)\right) \\
& \text { where } b^{\sim}(m)=\left\{\begin{array}{l}
b(m), m=0,1, \ldots, M-1 ; \\
R, m=M .
\end{array}\right.
\end{aligned}
$$

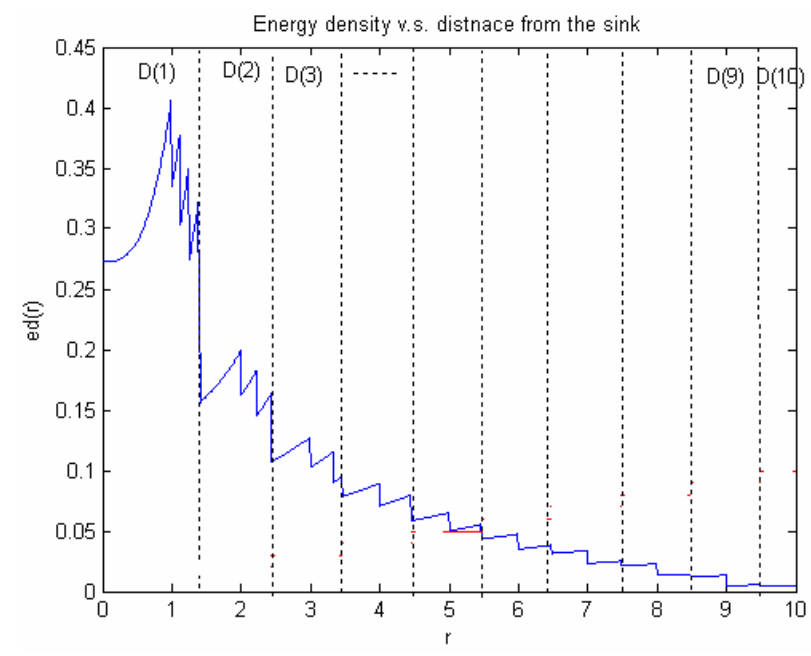

Figure 4. Energy density vs. distance from the sink.

We have performed several simulations to evaluate CSRT algorithm and to find energy density in a given deployment region. The system parameters selected for those simulations are: $T_{\min } / E_{0}=1, \mu=1, \beta=3, \alpha_{\mathrm{R}}=\alpha_{\mathrm{T} 0=} \alpha_{\mathrm{T}}=0.5, \alpha_{\mathrm{S}}=0$ and $R=10$. The energy density as a function of distance from the sink for the given system parameters is illustrated in the figure 4 . The figure shows that as we get close to the sink, inside each disc, the energy density has several peaks with increasing height. Also sharp density transitions occur at the boundary between discs.

In the next simulations we compare equation (15) and actual value of the total energy for different node densities. We simulated a Poisson distribution of sensor nodes in a circular region with the algorithm proposed in [17].In these simulations each sensor node finds its minimum energy path to the sink and sends its data through the nodes in that path to the sink for a period of time. At the end of simulations, total energy consumed by all nodes in the network was measured and is reported as the actual value of total energy. Figure 5 shows the result for a circular region. We did the same simulation for a collinear network and the result is reported in figure 6 . It must be noted that the simulations were run many times and the average results of those runs are reported in the figures. In the collinear case, as node density increases, the error between CSRT-obtained and simulated energy dissipations diminishes, however, in the circular case, a small difference remains throughout. In the following we attempt to improve these results. 


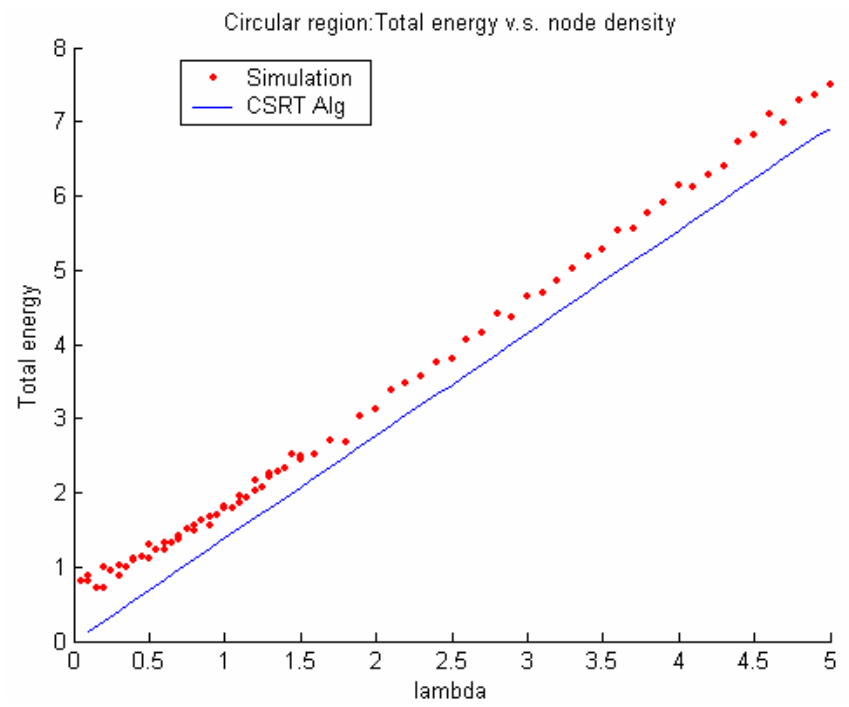

Figure 5. Actual value of total energy and total energy obtained by CSRT algorithm for a circular region in different node densities.

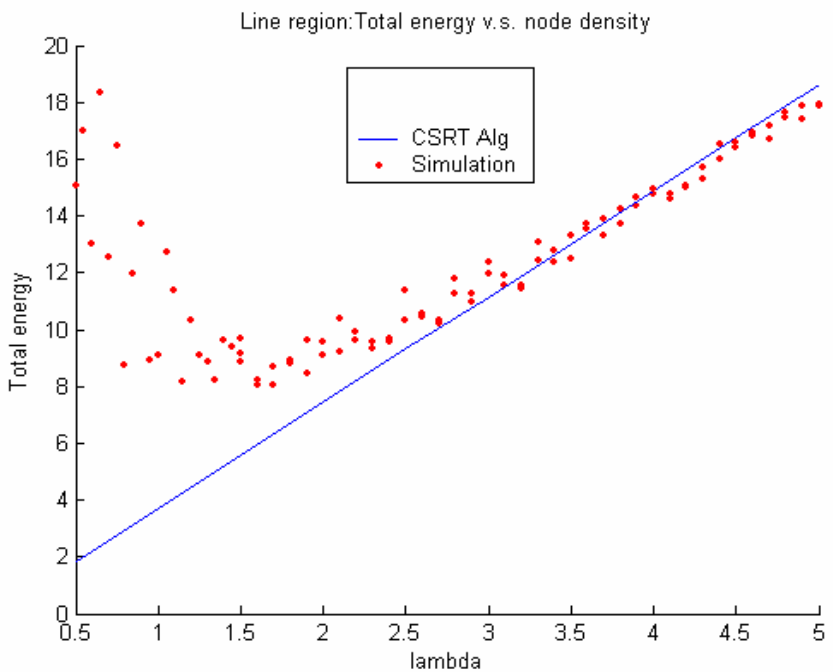

Figure 6. Actual value of total energy and total energy obtained by CSRT algorithm for a collinear network in different node densities.

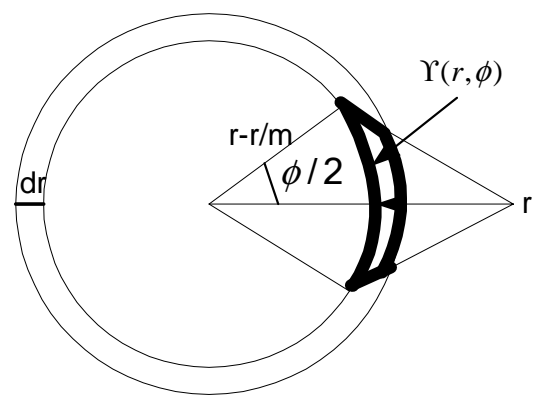

Figure 7. Area that a node located at distance $\mathrm{r}$ can find its predecessor (in $\Phi$ CSRT algorithm.)

\section{Analyzing CSRT and Modifying Routing Scheme}

First we analyze why the error in the circular case persists as $\lambda$ is increased. The CSRT algorithm assigns to each point $x=(r, \theta) \in D(m)$, a predecessor $x_{p}=(r-r / m, \theta)$ with the assumption that $\operatorname{Pr}\left(N\left(d x_{p}\right)=1 \mid N(d x)=1\right)=1$. This means that if a node is located in $(r, \theta)$, then with probability 1 another node can be found as its predecessor at location $(r-r / m \quad \theta)$.We call the conditional probability $h=\operatorname{Pr}\left(N\left(d x_{p}\right)=1 \mid N(d x)=1\right)$ as the hit probability. By using the property of exponential distribution, $h$ can be bounded as follows:

$$
h=\left\{\begin{array}{lc}
o(\lambda d r d \theta) \approx 0 \text { for the circular region } \\
o(\lambda d r) \quad \text { for the linear region }
\end{array}\right.
$$

Therefore, for the circular region, $h$ is bounded by a very small value even for large $\lambda$ values whereas, for the linear region, the upper bound on $h$ increases with $\lambda$.

Next we modify the CSRT algorithm to increase its hit probability. From equation (15), we must spend more energy in order to increase the hit probability. The modification is only in the way that the predecessor of a sensor node is identified. More precisely, for a point that is placed at $(r, \theta)$, instead of selecting a predecessor at point $(r-r / m, \theta)$, we randomly assign a predecessor at point $\left(r^{\prime}, \theta^{\prime}\right)$ in the following area(see figure 7 ):

$$
\begin{aligned}
& \Upsilon(r, \phi)=\left\{\left(r^{\prime}, \theta^{\prime}\right): r-r / m-\Delta r / 2 \leq r^{\prime}<r-r / m+\Delta r / 2\right. \\
& \left.\quad \text { and } \theta-\phi / 2 \leq \theta^{\prime}<\theta+\phi / 2\right\}
\end{aligned}
$$

where $\Delta r$ denotes the width of an infinitesimally small ring.

The hit probability of the modified algorithm is now bounded as follows:

$$
\operatorname{Pr}\left(N\left(d x_{p}\right)=1 \mid N(d x)=1\right)=o(\lambda \phi r d r)
$$

We call the modified algorithm as Ф-CSRT algorithm. Derivation of the minimum total energy in the network under this routing algorithm is straight-forward and is omitted here. We however report the total energy obtained by $\Phi$-CSRT for several values of $\Phi$ and compare them with the actual values of total energy obtained by simulation in figure 8. As can be seen, this time, the actual total energy converges to the value predicted by the $\Phi$-CSRT as $\lambda$ is increased.

\section{CONCLUSIONS}

In this paper, we considered the problem of energy efficient random deployment of sensor network. We found the sensor node density, or alternatively, the energy resource density at every point inside a given deployment region, which results in allocating the minimum total number of deployed sensors, or alternatively, the minimum total energy source subject to constraints on the QoM and network lifetime. As future work we plan to modify and improve the algorithms scheme given in this paper for sparse networks when the node density is small. 


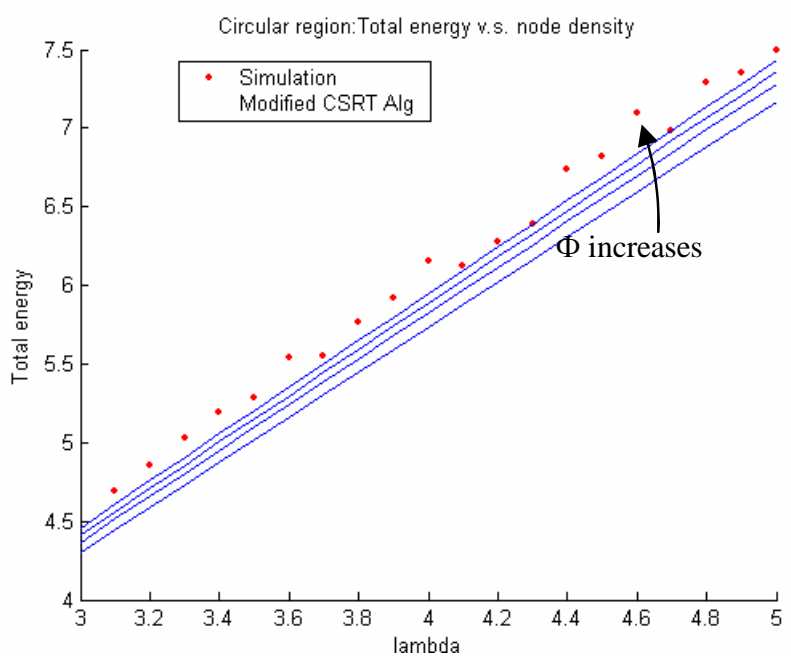

Figure 8. Actual total energy and the total energy obtained by $\Phi$-CSRT algorithm in different node densities and $\Phi$ values.

\section{REFERENCES}

1. S. Adlakha, S. Ganeriwal, C. Schurgers, and M. B. Srivastava, "Density, accuracy, latency and lifetime tradeoffs in wireless sensor networks - A multidimensional design perspective," Proc. of VTC: Symp. on Wireless Ad hoc, Sensors and Wearable Networks, Oct 2003.

2. H. Chang and L. Tassiulas, "Energy Conserving Routing in Wireless Ad Hoc Networks," Proc. of Infocom, pp. 22-31, Apr 2001.

3. M. Bhardwaj and A.P. Chandrakasan, "Bounding the Lifetime of Sensor Networks Via Optimal Role Assignments," Proc. of Infocom, pp. 15871596, New York, June 2002.

4. D. Ganesan, R. Cristescu and B. B-Lozano,'Power-Efficient Sensor Placement and Transmission Structure for Data Gathering under Distortion Constraints,"Proc. of Information Processing on Sensor Networks(IPSN),Apr 2004.

5. Y. Xu, J. Heidemann, and D. Estrin, "Geography-informed Energy Conservation for Ad Hoc Routing," Proc. of Mobile Computing and Networking (Mobicom ), pp. 70-84, July 2001.

6. S. Shakkottai, R. Srikant and N. Shroff," Unreliable Sensor Grids: Coverage, Connectivity and Diameter," Proc. of Infocom, Apr. 2003.

7. L. Booth, J. Bruck, M. Franceschetti and R. Meester," Covering Algorithms, Continuum Percolation, and the Geometry of Wireless Networks," Annals of Applied Probability, vol. 13,no.2, May 2003.

8. P. Hall., "Introduction to the Theory of Coverage Processes," John Wiley and Sons, 1988.

9. H. Zhang and J. Hou,"On Deriving the Upper Bound of $\alpha$-lifetime for Large Sensor Networks," Proc. of MobiHoc, May 2004.

10. R.Cristescu, B.B.Lozano and M.Vetterli,"On Network Correlated Data Gathering," Proc. of Infocom, Mar.2004.

11. M.Maleki and M.Pedram," Bounds of Average Spatial Distortion in Randomly Deployed Sensor Networks," Technical Report, CENG 04, EESystems, USC, Nov. 2004.

12. W. R. Heinzelman, A.Chandrakasan, and H. Balakrishnan, "EnergyEfficient Communication Protocol for Wireless Microsensor Networks," Proc. of the 33rd Annual Hawaii International Conference on System Sciences, pp. 3005-3014, Jan. 2000.

13. J. Elson and D. Estrin, "Time Synchronization for Wireless Sensor Networks," Proc. of IPDPS Workshop on Parallel and Distributed Computing Issues in Wireless Networks and Mobile Computing, Apr. 2001.

14. S. Ganeriwal, R. Kumar, M. B. Srivastava,"Timing-sync protocol for sensor networks," Proc. of the First ACM Conference on Embedded Networked Sensor Systems (SenSys),2003.
15. A.Savvides, C.C. Han, and M.B.Srivastava,"Dynamic Fine Grained Localization in Ad-Hoc Sensor Networks," Proc. of Mobicom, pp. 166179 , July 2001

16. M.Maleki and M.Pedram,"Continuous Space Model and Optimal Routing Algorithms for random Deployment of Sensor Networks," Technical Report, CENG 04, EE-Systems, USC, Nov. 2004.

17. S.M.Ross,"Introduction to Probability Models," Academic Press,Eighth Edition,2003.

18. V.Barnett,"Environmental Statistics: Methods and Applications," John Wiley and Sons, 2004. 